\title{
PRÁTICA DO ENFERMEIRO NO INCIDENTE COM MÚLTIPLAS VÍTIMAS
}

\section{NURSE'S PRACTICE IN THE INCIDENT WITH MULTIPLE VICTIMS}

\author{
Kályta Sabrina Monteiro de Sousa ${ }^{1}$ * Vanessa Rutiele Gomes de Sousa Martins ${ }^{2} *$ Izabel Maria $^{2}$ \\ Moreira Rodrigues $^{3} *$ Adriana Rodrigues Alves de Sousa ${ }^{4} *$ Filipe Augusto de Freitas Soares $5 *$ \\ Marcelo de Moura Carvalho ${ }^{6} *{\text { Rosana Serejo dos } \text { Santos }^{7} * \text { Maria Tamires Alves Ferreira }}^{8} *$ \\ Francineide Dutra Vieira9
}

\begin{abstract}
RESUMO
Objetivo: Analisar na literatura científica quais intervenções do enfermeiro no Incidente com Múltiplas Vítimas .Método trata-se de revisão integrativa de literatura, onde a busca dos estudos primários foi realizada nas bases de dados National Library of Medicine National Institutes of Health; Cummulative Index to Nursing and Allied Health Literature, Biblioteca Virtual em Saúde e Business Source Complete. na qual ambas totalizaram 391 artigos referente a temática e após a filtragem obedecendo os critérios de inclusão e exclusão utilizou-se 22 artigos para a elaboração deste projeto. Resultados: Para fornecer um atendimento de qualidade nas situações de desastres, faz-se necessário a preparação do enfermeiro através de simulação realísticas para uma melhor desenvoltura de competências e habilidades. Além de ser um instrumento válido para identificar falhas e avaliar a equipe. A troca de experiencia nesse contexto relacionadas a atuação tornase importante pois tem o intuito de aliviar o estresse e dá um preparo psicológico aos colegas em relação a temática. Conclusão: Portanto, o estudo sobre a atuação do enfermeiro em um IMV, mostrou que este profissional possui um papel crucial nesse contexto, pois este executa uma importante função no gerenciamento de recursos e na triagem das vítimas envolvidas. Porém existe uma deficiência na preparação destes, iniciando na graduação pela abordagem restrita da temática e pela falta de ensino simulado.
\end{abstract}

Palavras-chave: Enfermeiros e Enfermeiras. Cuidados de Enfermagem. Incidentes com Feridos em Massa.

\begin{abstract}
Objective: To analyze in the scientific literature which nurses' interventions in the Multiple Victim Incident (IMV). Method is an integrative literature review, where the search for primary studies was carried out in the databases National Library of Medicine National Institutes of Health; Cummulative Index to Nursing and Allied Health Literature, Virtual Health Library e Business Source Complete in which both totaled 391 articles related to the theme and after filtering obeying the inclusion and exclusion criteria using 22 articles for the elaboration of this project. Results: To provide quality care in the event of disasters, it is necessary to prepare the nurse through realistic simulation for a better resourcefulness of skills and abilities. In addition to being a valid instrument to identify failures and evaluate a team. The exchange of experience in this context related to performance becomes important because it is intended to relieve stress and provides psychological preparation to colleagues in relation to the theme. Conclusion: Therefore, the study on the role of nurses in an IMV, revealed that this professional has a crucial role in this context, as he performs an important function in the management of resources and in the screening of the victims involved. However, there is a deficiency in the preparation of these, starting at graduation due to the restricted approach of the theme and the lack of simulated teaching.
\end{abstract}

Keywords: Nurses and Nurses. Nursing Care. Mass Incidents.

\footnotetext{
${ }^{1}$ Faculdade Estácio de Teresina - Teresina- Brasil. ORCID: https://orcid.org/0000-0003-1248-9213

${ }^{2}$ Faculdade Estácio de Teresina - Teresina- Brasil. ORCID https://orcid.org/0000-0002-6329-7767

${ }^{3}$ Faculdade Estácio de Teresina - Teresina- Brasil. ORCID https://orcid.org/0000-0002-0579-6226

${ }^{4}$ Faculdade Estácio de Teresina - Teresina- Brasil. ORCID https://orcid.org/0000-0003-3245-443X

${ }^{5}$ Faculdade Estácio de Teresina - Teresina- Brasil. ORCID https://orcid.org/0000-0003-0510-3968

${ }^{6}$ Faculdade Estácio de Teresina - Teresina- Brasil. ORCID https://orcid.org/0000-0003-4322-404X

${ }^{7}$ Faculdade Estácio de Teresina - Teresina- Brasil. ORCID https://orcid.org/0000-0003-3084-7539

${ }^{8}$ Faculdade Estácio de Teresina - Teresina- Brasil. ORCID: https://orcid.org/0000-0001-7822-9575

${ }^{9}$ Faculdade Estácio de Teresina - Teresina- Brasil. ORCID: https://orcid.org/0000-0001-9540-999X
} 


\section{INTRODUÇÃO}

A Organização Mundial de Saúde (OMS) designa incidente com múltiplas vítimas (IMV) um evento que produz sincronicamente admiráveis números de vítimas, podendo atrapalhar a assistência atribuída rotineiramente ${ }^{(1)}$. No Brasil, o Ministério da Saúde conceptualiza um IMV, quando este envolve um número superior ou igual a cinco vítimas ${ }^{(2)}$.

As situações que abrangem múltiplas vítimas concomitantemente, acarretam um grande impacto social, pois em sua maioria incluem perdas humanas, materiais $\mathrm{e}$ ambientais $^{(3)}$ demandando uma administração adequada do incidente, programação e organização sistemática dos serviços de urgência, de forma que assegure a oferta apropriada, e evite o ônus dos serviços de saúde juntamente com a necessidade de uma demanda brusca por profissionais, equipamentos e estruturas hospitalares para o atendimento imediato ${ }^{(4)}$.

$\mathrm{O}$ enfermeiro tem sua atuação respaldada na lei $\mathrm{n}^{\mathrm{o}} 7498$, de 25 de junho de 1986, que define como atividade própria deste profissional a assistência direta ao paciente grave, a execução de atividades de maior complexidade técnica, que requerem conhecimento de base científica e habilidade de tomar decisão ágil. Por se tratar de salvar vidas, é imprescindível que o profissional desempenhe trabalhos certeiros e tenha capacidade para cumprir uma atividade adequada diante um $\mathrm{IMV}^{(5)}$.

Os incidentes com múltiplas vítimas possuem etapas de regulamentação médicas distintas. Em situações como essas, o atendimento pré-hospitalar realizado possui fundamentos bem distintos, pois quando se trata de a uma única vítima, todo recurso deve ser oferecido na tentativa de salvar a vida ou evitar consequências no indivíduo. Em Incidentes de Múltiplas Vítimas (IMV) se exige a tentativa de salvar o maior número de vítimas possíveis. Surgindo então a necessidade de estabelecer prioridades através da triagem ${ }^{(6)}$.

Para que qualquer atendimento préhospitalar aconteça de forma competente, é essencial que a primeira equipe que chegue ao local organize a segurança da cena, e faça a demarcação das chamadas zona quente, zona morna e zona fria, assim facilitando o atendimento as vítimas ${ }^{(7)}$. $\mathrm{O}$ êxito do atendimento em um IMV está na preparação dos profissionais; disponibilidade de recursos; habilidade e agilidade da equipe ${ }^{(8,9)}$.

Apesar de não existir estatísticas que apresentam a proporção real dos Incidentes com Múltiplas Vítimas no nosso cenário sanitário, fica evidente que esses se manifestam contemporaneamente como problemas corriqueiros, nos quais os acidentes de trânsito, unidos com as inundações bruscas, deslizamentos de terra, rompimento de barragens e outros desastres 
naturais configuram-se como consideráveis etiologias para esses eventos ${ }^{(10)}$.

Os IMV estão se tornando cada vez mais comuns, podendo causar desordens para o sistema de saúde, que necessita de profissionais qualificados. $\mathrm{O}$ enfermeiro tem função fundamental na assistência, por isso é necessário apresentar conhecimentos e habilidades para fornecer um atendimento de qualidade e diminuir erros ${ }^{(11)}$. Para que isso ocorra é necessário a capacitação e treinamento para adquirir e aperfeiçoar competências. Desse modo, é necessário o aprimoramento precedente dos profissionais, ainda no ambiente da graduação $^{(4)}$, pois o tema é abordado de forma vaga durante os cursos de enfermagem, gerando dúvidas aos profissionais recém formados para a atuação na área.

Em virtude dos fatos mencionados anteriormente, surge o objetivo deste estudo analisar na literatura científica quais intervenções do enfermeiro no Incidente com Múltiplas Vítimas (IMV).

\section{METODOLOGIA}

Trata-se de uma revisão integrativa de literatura, pois é um método que tem como finalidade sintetizar resultados obtidos em pesquisas sobre um tema ou questão, de maneira sistemática, ordenada e abrangente. É denominada integrativa porque fornece informações mais amplas sobre um assunto/problema, constituindo, assim, um corpo de conhecimento. Esse método permite a inclusão simultânea de pesquisa quaseexperimental e experimental, combinando dados de literatura teórica e empírica, proporcionando compreensão mais completa do tema de interesse $\mathrm{e}^{(12)}$.

Para a elaboração da questão de pesquisa da revisão integrativa, utilizou-se a estratégia PICo (P - população; I intervenção/área de interesse; Co - contexto) onde "P" é enfermeiros; "I" conhecimentos e habilidades do enfermeiro e $\mathrm{O}$ " $\mathrm{Co}$ " atendimento pré-hospitalar. $\mathrm{O}$ uso dessa estratégia para formular a questão de pesquisa na condução de métodos de revisão possibilita a identificação de palavras-chave, as quais auxiliam na localização de estudos primários relevantes nas bases de dados ${ }^{(13)}$. Assim, a questão de pesquisa construída foi: $\mathrm{O}$ que a literatura traz sobre a prática enfermeiro no atendimento pré-hospitalar em um incidente com múltiplas vítimas?

O levantamento bibliográfico ocorreu nas seguintes bases de dados: National Library of Medicine National Institutes of Health (PubMed); Cummulative Index to Nursing and Allied Health Literature (CINAHL), Biblioteca Virtual em Saúde (BVS/Bireme) e Business Source Complete (EBSCO). Para a busca dos estudos primários nas respectivas bases de dados, foram utilizados os descritores controlados Descritores em Ciência da Saúde (Decs), 
Quadro 1 - Termos de busca e descritores guiados pelo acrônimo PICo nas bases de dados.

\begin{tabular}{|l|l|l|}
\hline PICo & TERMOS DE BUSCA & TIPO \\
\hline P & $\begin{array}{l}\text { Enfermeiras e Enfermeiros/ Nurses/ } \\
\text { Enfermeras y Enfermeros }\end{array}$ & $\begin{array}{l}\text { DECS } \\
\text { MESH } \\
\text { DESCRITOR EM ESPANHOL }\end{array}$ \\
\hline I & $\begin{array}{l}\text { Cuidados de Enfermagem/ Cuidados } \\
\text { de Enfermagem/ } \\
\text { Atención de Enfermería/ Protocolos/ } \\
\begin{array}{l}\text { Protocols/ Protocolos/Conhecimento/ } \\
\text { Knowledge/Conocimiento }\end{array}\end{array}$ & $\begin{array}{l}\text { DESC } \\
\text { MESH } \\
\text { DESCRITORES EM ESPANHOL }\end{array}$ \\
\hline Co & $\begin{array}{l}\text { Incidentes com Feridos em Massa/ } \\
\text { Mass Casualty Incidents/Incidentes } \\
\text { con Víctimas en Masa/Acidentes com } \\
\text { Múltiplas Vítimas/Grande Número de } \\
\text { Feridos }\end{array}$ & $\begin{array}{l}\text { DECS } \\
\text { MESH } \\
\text { DESCRITORES EM ESPANHOL } \\
\text { DESCRITORES NÃO } \\
\text { CONTROLADOS }\end{array}$ \\
\hline
\end{tabular}

Fonte: Dados da Pesquisa, 2020.

Legenda: DeCS: Descritores em Ciências da Saúde.

Foram incluídos na pesquisa artigos originais disponíveis na íntegra e online nas bases de dados selecionadas, publicados nos idiomas português, inglês e espanhol, no período de 2010 a 2020 e que aborde a temática do estudo. Como critério de exclusão artigos que estiverem duplicados nas bases de dados, artigos em que não haja relação com o contexto do tema proposto por meio da inspeção do título e resumo, publicações do tipo editorial, dissertações, revisões de literatura. Em cada base de dados foi utilizada, uma estratégia de busca específica e a seleção dos dados foi realizada por três revisores de forma independente, com o objetivo de averiguar com mais precisão os artigos.

Quadro 2 - Estratégia de busca dos artigos nas bases de dados.

\begin{tabular}{|l|l|}
\hline BASE DE DADOS & ESTRATÉGIA DE BUSCA \\
\hline CINAHL & $\begin{array}{l}\text { Nurses AND Nursing care OR knowledge AND Mass casualty incident } \\
\text { OR mass casualty event. }\end{array}$ \\
\hline PUBMED & Nurses AND Nursing care OR knowledge AND Mass casualty incidents \\
\hline BVS & $\begin{array}{l}\text { Enfermeiras e enfermeiros OR Nuses OR Enfermeras y Enfermeros } \\
\text { AND Cuidados de enfermagem OR nursing care OR Atención de } \\
\text { enfermería OR rotocolos OR conhecimento OR Knowledge OR } \\
\text { Conocimiento AND Incidente com feridos em massa OR mass casualty } \\
\text { incidents OR incidentes con victimas en masa OR acidentes com } \\
\text { multilas vitimas OR grande número de feridos OR Feridos Em Massa. }\end{array}$ \\
\hline EBSCO & $\begin{array}{l}\text { Enfermeiras e enfermeiros OR Nuses OR Enfermeras y Enfermeros } \\
\text { AND Cuidados de enfermagem OR nursing care OR Atención de } \\
\text { enfermería OR protocolos OR conhecimento OR Knowledge OR }\end{array}$ \\
\hline
\end{tabular}


Conocimiento AND Incidente com feridos em massa OR mass casualty incidents OR incidentes con victimas en masa OR acidentes com múltiplas vítimas OR grande número de feridos.

Fonte: Elaborado pelos autores, 2020.

Após leitura minuciosa dos títulos e resumos, ao refinarmos a pesquisa, os números de artigos encontrados, excluídos e selecionados por bases de dados foram organizados no fluxograma 1 , evidenciando o quantitativo total de artigos encontrados, de excluídos e os que ficaram de amostra final do estudo.

Fluxograma 1 - Seleção dos artigos para a revisão integrativa da literatura, elaborado a partir das recomendações PRISMA.

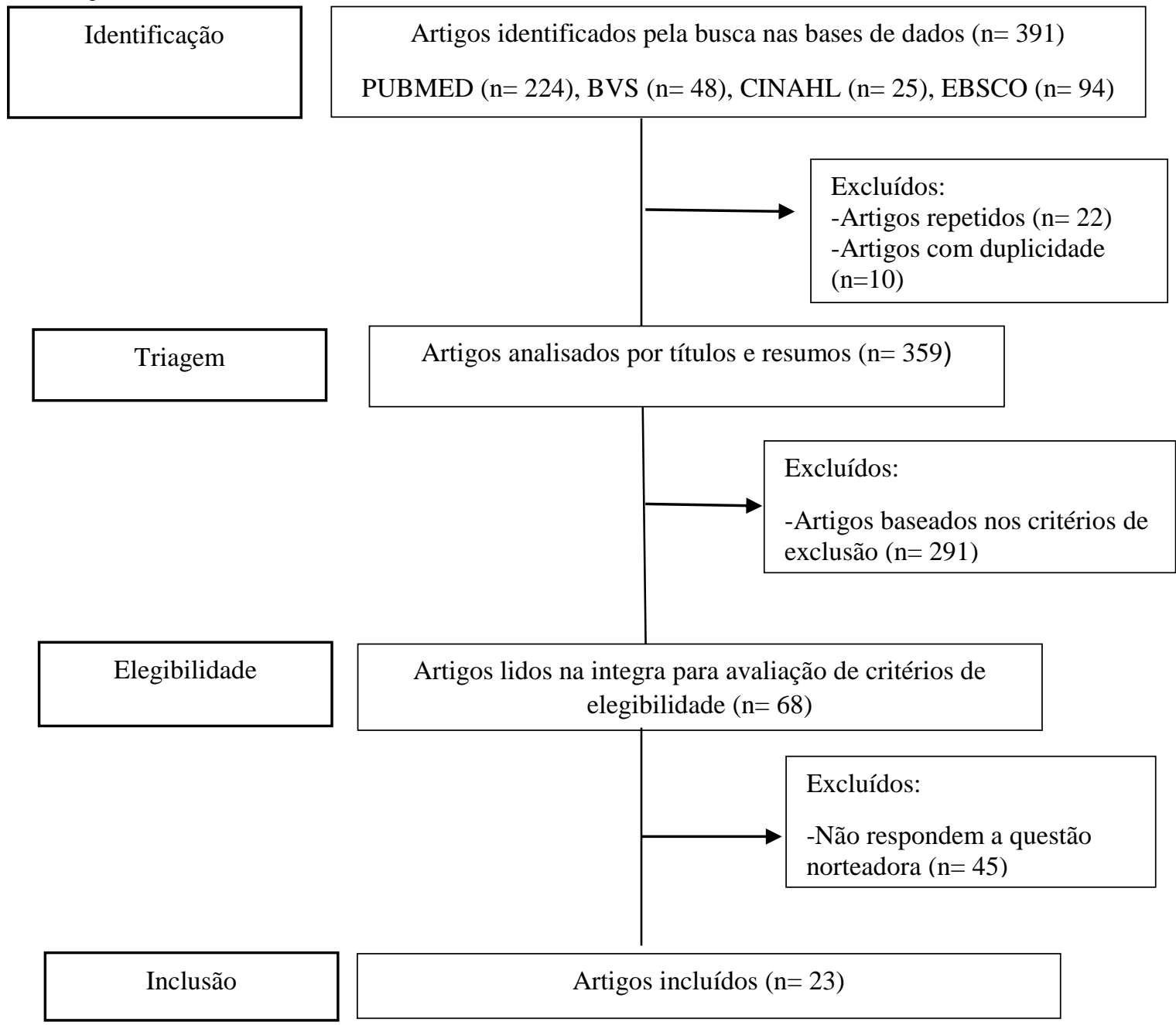

Fonte: Elaborado pelos autores, 2020.

$\mathrm{Na}$ etapa de avaliação crítica dos estudos selecionados, os artigos foram organizados em uma tabela de forma descritiva, na qual será realizada a comparação dos resultados dos artigos científicos selecionados. Registraram-se os seguintes aspectos dos artigos escolhidos: identificação do estudo (autores, ano de publicação, título do periódico, título do artigo e nível de evidência científica) e 
características metodológicas do estudo e os achados referentes aos conhecimentos e habilidades do enfermeiro no IMV os artigos foram organizados em uma tabela de forma descritiva, na qual será realizada a comparação dos resultados dos artigos científicos selecionados.

O nível de evidência dos estudos baseou-se na classificação das forças de evidência, segundo os autores ${ }^{(14)}$, que determina as evidências em seis níveis, sendo: Nível 1: evidências resultantes da metaanálise de múltiplos estudos clínicos controlados e randomizados; Nível 2: evidências obtidas em estudos individuais com delineamento experimental; Nível 3: evidências de estudos quase-experimentais; Nível 4: evidências de estudos descritivos (não-experimentais) ou com abordagem qualitativa; Nível 5: evidências provenientes de relatos de caso ou de experiência; Nível 6: evidências baseadas em opiniões de especialistas.

\section{RESULTADOS}

No Quadro 3, são apresentados os resultados das publicações quanto as características, à autoria do estudo, ano de publicação, país de origem, título, periódico, delineamento de pesquisa, nível de evidência e conhecimentos e habilidades do enfermeiro no IMV.

Quadro 3 - Caracterização conhecimentos e habilidades do enfermeiro em incidente com múltiplas vítimas.

\begin{tabular}{|c|c|c|c|c|c|}
\hline Autor & $\begin{array}{l}\text { Ano/ } \\
\text { País }\end{array}$ & Periódico & $\begin{array}{l}\text { Delineamento } \\
\text { da pesquisa* }\end{array}$ & $\begin{array}{c}\text { Nível de } \\
\text { Evidência*** }\end{array}$ & $\begin{array}{c}\begin{array}{c}\text { Conhecimentos e habilidades } \\
\text { do enfermeiro }\end{array} \\
\end{array}$ \\
\hline $\begin{array}{l}\text { Busby, } \\
\text { Witucki- } \\
\text { Brown. } \\
\text { (15) }\end{array}$ & $\begin{array}{l}2010 \\
\text { EUA }\end{array}$ & $\begin{array}{l}\text { Journal of } \\
\text { Emergency } \\
\text { Nursing }\end{array}$ & $\begin{array}{l}\text { Abordagem } \\
\text { qualitativa }\end{array}$ & IV & $\begin{array}{l}\mathrm{O} \text { artigo fala sobre a importância } \\
\text { do gerenciamento através da } \\
\text { preparação. }\end{array}$ \\
\hline $\begin{array}{l}\text { Jong, } \\
\text { Benner, } \\
\text { Benner }^{(16)}\end{array}$ & $\begin{array}{c}2010 \\
\text { Iraque }\end{array}$ & $\begin{array}{l}\text { Journal of } \\
\text { trauma } \\
\text { nursing }\end{array}$ & $\begin{array}{l}\text { Abordagem } \\
\text { qualitativa }\end{array}$ & IV & $\begin{array}{l}\text { Por meio da troca de } \\
\text { experiências pode-se obter } \\
\text { novos conhecimentos além de } \\
\text { desenvolver habilidades } \\
\text { refletir sobre suas ações }\end{array}$ \\
\hline Sloan $^{(17)}$ & $\begin{array}{l}\text { 2010/ } \\
\text { Estados } \\
\text { Unidos }\end{array}$ & $\begin{array}{l}\text { Journal of } \\
\text { Emergency } \\
\text { Nursing }\end{array}$ & $\begin{array}{l}\text { Relatos de } \\
\text { experiência }\end{array}$ & $\mathrm{V}$ & $\begin{array}{l}\text { O artigo fala sobre a } \\
\text { necessidade de treinamentos } \\
\text { para um bom preparo } \\
\text { enfatizando o compartilhamento } \\
\text { por meio de experiências. }\end{array}$ \\
\hline $\begin{array}{l}\text { Dasgupta } \\
\text { et } \mathrm{al}^{(18)}\end{array}$ & $\begin{array}{c}2011 / \\
\text { Jamaica } \\
\text { (América } \\
\text { Central) }\end{array}$ & $\begin{array}{l}\text { Prehospital } \\
\text { and Disaster } \\
\text { Medicine }\end{array}$ & $\begin{array}{l}\text { Relatos de } \\
\text { experiência }\end{array}$ & $\mathrm{V}$ & $\begin{array}{l}\text { A preparação faz-se necessária } \\
\text { por meio da preparação de } \\
\text { simulações de triagem. }\end{array}$ \\
\hline $\begin{array}{l}\text { Admi et } \\
\mathrm{al}^{(19)}\end{array}$ & $\begin{array}{l}2011 \\
\text { Israel }\end{array}$ & $\begin{array}{l}\text { Jornal of } \\
\text { nursing } \\
\text { scholarship }\end{array}$ & $\begin{array}{l}\text { Pesquisa } \\
\text { descritiva }\end{array}$ & IV & $\begin{array}{l}\text { Artigo fala sobre } \\
\text { gerenciamento de } \\
\text { qual seu preparo é fundamental. }\end{array}$ \\
\hline Glow et & $2013 /$ & Medicina & Estudo & II & Fala sobre o gerenciamento do \\
\hline
\end{tabular}




\begin{tabular}{|c|c|c|c|c|c|}
\hline al. ${ }^{(20)}$ & $\begin{array}{l}\text { Estados } \\
\text { Unidos } \\
\end{array}$ & $\begin{array}{l}\text { pré-hospitalar } \\
\text { e de desastres }\end{array}$ & experimental & & $\begin{array}{llll}\begin{array}{l}\text { enfermeiro } \\
\text { preparação }\end{array} & \text { por meio da } \\
\end{array}$ \\
\hline$\underset{(21)}{\text { Shannon, }}$ & $\begin{array}{l}2015 / \\
\text { Estados } \\
\text { Unidos }\end{array}$ & $\begin{array}{l}\text { Journal of } \\
\text { Nursing } \\
\text { Education }\end{array}$ & $\begin{array}{l}\text { Relato de } \\
\text { experiência }\end{array}$ & $\mathrm{V}$ & $\begin{array}{l}\text { O artigo fala sobre preparação e } \\
\text { seus benefícios }\end{array}$ \\
\hline $\begin{array}{l}\text { Fletcher, } \\
\text { et al }{ }^{(22)}\end{array}$ & $\begin{array}{c}\text { 2015/ } \\
\text { Estados } \\
\text { Unidos }\end{array}$ & $\begin{array}{l}\text { journal of } \\
\text { trauma } \\
\text { nursing }\end{array}$ & $\begin{array}{l}\text { Relato de } \\
\text { experiência }\end{array}$ & $\mathrm{V}$ & $\begin{array}{l}\mathrm{O} \text { artigo fala sobre preparação e } \\
\text { seus benefícios. }\end{array}$ \\
\hline $\begin{array}{l}\text { VandenBer } \\
\text { g, e Scott. }\end{array}$ & $\begin{array}{c}\text { 2015/ } \\
\text { Estados } \\
\text { Unidos }\end{array}$ & $\begin{array}{l}\text { Critical Care } \\
\text { Nursing } \\
\text { Clinics of } \\
\text { North } \\
\text { America } \\
\end{array}$ & $\begin{array}{l}\text { Estudo } \\
\text { transversal }\end{array}$ & IV & $\begin{array}{l}\text { Fala sobre a preparação } \\
\text { essencial para um } \\
\text { planejamento }\end{array}$ \\
\hline $\begin{array}{c}\text { Campion et } \\
\mathrm{al}^{(24)}\end{array}$ & $\begin{array}{l}2016 / \\
\text { Estados } \\
\text { Unidos }\end{array}$ & $\begin{array}{l}\text { JAMA } \\
\text { Internal } \\
\text { Medicine }\end{array}$ & $\begin{array}{c}\text { Pesquisa } \\
\text { experimental }\end{array}$ & II & $\begin{array}{l}\text { Necessita ter conhecimento e } \\
\text { preparação para o planejamento } \\
\text { e gerenciamento de recursos } \\
\text { adequados. }\end{array}$ \\
\hline $\begin{array}{l}\text { Veenema } \\
\text { et } \mathrm{al}^{(25)}\end{array}$ & $\begin{array}{l}2017 \\
\text { EUA }\end{array}$ & Nurs admin $q$ & $\begin{array}{l}\text { Abordagem } \\
\text { mista } \\
\text { qualitativa } \\
\text { quantitativa } \\
\end{array}$ & IV & $\begin{array}{l}\mathrm{O} \text { artigo fala sobre os principais } \\
\text { requisitos do enfermeiro para } \\
\text { responder um IMV }\end{array}$ \\
\hline $\begin{array}{l}\text { Hammad } \\
\text { et } \mathrm{al}^{(26)}\end{array}$ & $\begin{array}{l}\text { 2017/ } \\
\text { Austráli } \\
\text { a }\end{array}$ & $\begin{array}{l}\text { Australasian } \\
\text { emergency } \\
\text { nursing jornal }\end{array}$ & $\begin{array}{c}\text { Abordagem } \\
\text { qualitativa }\end{array}$ & IV & $\begin{array}{l}\text { Preparação do enfermeiro e } \\
\text { importante para adquirir } \\
\text { conhecimentos e experiências. }\end{array}$ \\
\hline $\begin{array}{l}\text { Tseng et } \\
\mathrm{al}^{(27)}\end{array}$ & $\begin{array}{l}\text { 2017/ } \\
\text { Ásia }\end{array}$ & $\begin{array}{l}\text { Journal of } \\
\text { Burn Care \& } \\
\text { Research } \\
\end{array}$ & $\begin{array}{l}\text { Estudo } \\
\text { transversal }\end{array}$ & IV & $\begin{array}{l}\text { Necessário preparação } \\
\text { psicológica para desastres. }\end{array}$ \\
\hline $\begin{array}{c}\text { Jonson et } \\
\mathrm{al}^{(28)}\end{array}$ & $\begin{array}{l}2017 / \\
\text { Suécia }\end{array}$ & $\begin{array}{l}\text { Nurse } \\
\text { education } \\
\text { today }\end{array}$ & $\begin{array}{c}\text { Pesquisa } \\
\text { Experimental }\end{array}$ & II & $\begin{array}{l}\begin{array}{l}\text { Simulação é essencial para } \\
\text { desenvolver } \\
\text { gerenciamento e autoeficácia }\end{array} \\
\text { hobilidades }\end{array}$ \\
\hline $\begin{array}{l}\text { kiani et } \\
\mathrm{al}^{(29)}\end{array}$ & $\begin{array}{c}\text { Eua } \\
2017\end{array}$ & $\begin{array}{l}\text { Medicine, } \\
\text { Health Care } \\
\text { and } \\
\text { Philosophy }\end{array}$ & $\begin{array}{c}\text { Pesquisa } \\
\text { qualitativa }\end{array}$ & IV & $\begin{array}{l}\text { Conhecimento clínico } \mathrm{e} \\
\text { operacional assim como os de } \\
\text { gerenciamento de recursos e } \\
\text { saber a sua autoeficácia. }\end{array}$ \\
\hline $\begin{array}{l}\text { Verheul } \\
\text { et } \mathrm{al}^{(30)}\end{array}$ & $\begin{array}{c}\text { 2018/ } \\
\text { Holanda }\end{array}$ & $\begin{array}{l}\text { Prehospital } \\
\text { and Disaster } \\
\text { Medicine } \\
\end{array}$ & \begin{tabular}{|l|} 
Estudo \\
descritivo \\
retrospectivo \\
\end{tabular} & IV & $\begin{array}{l}\text { A preparação é essencial pois } \\
\text { através dela pode-se treinar, } \\
\text { avaliar e identificar lacunas. }\end{array}$ \\
\hline $\begin{array}{l}\text { McElroy } \\
\text { et } \mathrm{al}^{(31)}\end{array}$ & $\begin{array}{l}\text { 2019/ } \\
\text { Estados } \\
\text { Unidos } \\
\end{array}$ & Sugery & $\begin{array}{l}\text { Relato de } \\
\text { experiencia }\end{array}$ & $\mathrm{V}$ & $\begin{array}{l}\text { O exercício de simulações e uma } \\
\text { oportunidade para melhorias } \\
\text { além de avaliar protocolos. }\end{array}$ \\
\hline Shannon $^{(32)}$ & $\begin{array}{l}2019 \\
\text { INDIA }\end{array}$ & $\begin{array}{l}\text { Nurse } \\
\text { educator }\end{array}$ & $\begin{array}{c}\text { Pesquisa } \\
\text { experimental }\end{array}$ & II & 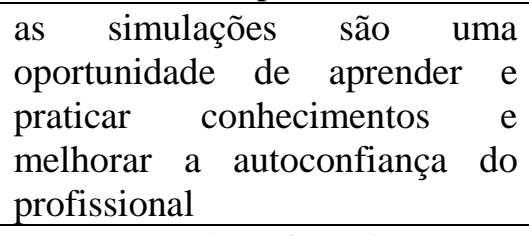 \\
\hline $\begin{array}{c}\text { Bentley et } \\
\mathrm{al}^{(33)}\end{array}$ & $\begin{array}{c}\text { 2019/ } \\
\text { Estados } \\
\text { Unidos }\end{array}$ & $\begin{array}{l}\text { MedEDPOR } \\
\text { TAL }\end{array}$ & $\begin{array}{c}\text { Pesquisa } \\
\text { experimental }\end{array}$ & II & $\begin{array}{l}\text { Preparação do enfermeiro e seus } \\
\text { benefícios e uma oportunidade } \\
\text { para gerenciar recursos }\end{array}$ \\
\hline $\begin{array}{l}\text { Kim e } \\
\operatorname{Lee}^{(34)}\end{array}$ & $\begin{array}{l}2020 / \\
\text { Coreia } \\
\text { do Sul }\end{array}$ & $\begin{array}{l}\text { Nurse } \\
\text { Education } \\
\text { Today }\end{array}$ & \begin{tabular}{|c} 
Estudo \\
descritivo, com \\
abordagem \\
quantitativa não \\
experimental
\end{tabular} & IV & $\begin{array}{l}\text { Fala sobre a preparação e os } \\
\text { benefícios e oportunidades para } \\
\text { uma autoavaliação. }\end{array}$ \\
\hline
\end{tabular}




\begin{tabular}{|c|c|l|c|c|c|}
\hline $\begin{array}{c}\text { Silva et } \\
\text { al }{ }^{(35)}\end{array}$ & $\begin{array}{c}2020 \\
\text { Brasil }\end{array}$ & $\begin{array}{l}\text { Revista } \\
\text { baiana de } \\
\text { enfermagem }\end{array}$ & $\begin{array}{c}\text { Estudo } \\
\text { descritivo, com } \\
\text { abordagem } \\
\text { quantitativa }\end{array}$ & IV & $\begin{array}{l}\text { Conhecimentos de } \\
\text { gerenciamentos e triagem, } \\
\text { pensamento crítico, capacitação } \\
\text { para isso a simulação tem uma } \\
\text { função muito importante no } \\
\text { desenvolvimento desses } \\
\text { conhecimentos e habilidades. }\end{array}$ \\
\hline $\begin{array}{c}\text { Screws e } \\
\text { Cason }\end{array}$ & $\begin{array}{c}\text { (37) } \\
\text { Estados } \\
\text { Unidos }\end{array}$ & $\begin{array}{l}\text { Nursing } \\
\text { education } \\
\text { perspectives }\end{array}$ & $\begin{array}{c}\text { Relato de } \\
\text { experiência }\end{array}$ & II & $\begin{array}{l}\text { Preparação por meio de } \\
\text { simulaçôes e seus benefícios }\end{array}$ \\
\hline
\end{tabular}

Fonte: Elaborado pelos autores, 2020.

*Os delineamentos enumerados neste quadro estão descritos como no artigo analisado.

** O nível de evidencia dos estudos foi determinado segundo classificação de Polit e Beck (2019)

Pela análise dos artigos selecionados, a grande parte dos estudos foi encontrada na base de dados PubMed $(69,56 \%)$, com maior concentração e maior frequência nos últimos dez anos, uma vez que a partir de 2010 foram encontrados mais artigos e com menor intervalo de tempo de publicação entre um e outro, com predomínio no ano de 2017 e 2019 (representando 21,73 e $17,39 \%$ respectivamente).

Estes dados podem estar relacionados com a preparação da triagem, conhecimento do enfermeiro em incidentes com múltiplas vítimas e gerenciamento de recursos e o trabalho em equipe. Os Estados Unidos lideraram no número de publicações $(60,86 \%)$. Dois estudos foram produzidos no Brasil (8,69\%). Nos países Iraque, Jamaica, Israel, Austrália, Suécia, Holanda e índia foi encontrado um artigo de cada país (4,34\%). a evidenciar a distribuição das pesquisas em todo mundo, em razão do complexo contexto de atuação do enfermeiro perante IMV, evidenciando a necessidade de preparação profissional para um conhecimento adequado de gerenciamento de recursos e habilidades que faz sua atuação ser eficaz diante a assistência as vítimas.

Quanto ao delineamento da pesquisa, evidenciou-se: estudo com abordagem qualitativa, evidência proveniente de relato de caso ou experiencia, pesquisa descritiva, estudo experimental, pesquisa participante, pesquisa de campo, estudo transversal e abordagem mista.

Em relação às forças de evidências observou-se que 8 artigos $(34,78 \%$ das publicações) com nível II, 10 artigos (43,47\%) possuem o nível de evidência IV, seguidas de 5 artigos $(21,73 \%)$ com nível de evidência V. Não foi encontrado nas publicações, trabalhos com nível de evidência I e III e VI

Em relação aos tipos de prática do enfermeiro em IMV faz-se necessário uma preparação eficaz através de meio de simulação é essencial para desenvoltura de competências relacionadas a atuação do enfermeiro, além de ser um instrumento válido para detectar lacunas e avaliar a 
equipe. A troca de experiencia faz-se importante pois tem o intuito de aliviar $\mathrm{o}$ estresse e dá um preparo psicológico aos colegas em relação ao contexto

Para melhor discutir os estudos analisados, optou-se pela organização dos artigos agrupados em três categorias: a categoria Habilidades e conhecimentos do enfermeiro no IMV, O papel da educação permanente através das simulações realísticas no IMV e Triagem no contexto de incidente com múltiplas vítimas.

\section{DISCUSSÃO}

$\mathrm{Na}$ ocorrência de episódios envolvendo muitas vítimas, o trabalho da enfermagem nos cuidados críticos é indispensável, entretanto vai depender das estruturas institucionais, recursos disponíveis e quantidade de profissionais participantes no atendimento às vítimas ${ }^{(11)}$. Algumas exigências gerais são consideráveis para que o enfermeiro exerça sua função de maneira apropriada sendo elas: organização, percepção, e habilidade de tomar decisões ágeis, especificar prioridades, avaliar o paciente em uma abordagem integral e executar intervenções quando preciso, juntamente com a equipe médica ${ }^{(3)}$.

\section{Habilidades e conhecimentos do enfermeiro no IMV}

O enfermeiro deve possuir habilidades em triagem, um bom gerenciamento de recursos, além de um preparo psicológico ${ }^{(36)}$. Os conhecimentos para uma boa atuação do enfermeiro incluem aspecto de liderança, comunicação, raciocínio clínico, autoconfiança, habilidades cognitivas, psicomotoras, atitudinais e conhecimento para tratar vítimas politraumatizadas sobretudo ser cooperativo, realista, acessível e receptivo aos feedbacks ${ }^{(25)}$. Para uma atuação de qualidade o enfermeiro deve possuir conhecimentos básicos de emergências e o processo de triagem corretamente ${ }^{(21)}$.

Os enfermeiros desempenham papéis significativos em todos os níveis de gerenciamento em um incidente com múltiplas vítimas, pois coordenam e supervisionam a assistência contribuindo no tratamento e recuperação da saúde, responsabilizando-se pela garantia da qualidade e continuidade do cuidado ${ }^{(19)}$.

Os autores ${ }^{(28)}$. diz o enfermeiro tem uma importante atuação no gerenciamento de recursos para o atendimento adequado e que a superlotações e a constante falta dos recursos apropriados influencia diretamente no atendimento pois reduz a capacidade de resposta ao surto que exige ativação de protocolos de desastre e o uso ideal de todos os recursos disponíveis no momento.

Ainda nesse contexto os autores ${ }^{(23)}$, afirmam que o enfermeiro tem um papel importante no gerenciamento de recursos, 
através de ações junto com equipe medica diante de um incidente com múltiplas vítimas, como busca de recursos para o atendimento as vítimas além de evitar enxurrada de famílias ou amigos que procuram o hospital em busca informações criando um estabelecimento para repasse das mesmas, evitando a aglomeração nas instituições de saúde. Importante lembrar que os enfermeiros são profissionais capacitados, para explicar a condição do paciente e dar suporte psicólogos às famílias das vítimas

Os autores ${ }^{(20)}$ relata que o enfermeiro dever saber sobre os gerenciamentos de recursos, devem ser capazes de administrar e prevenir as situações de crise de forma eficaz e gerenciar recursos disponíveis da equipe, equipamentos, espaço e preparação do hospital, o gerenciamento adequado requer liderança, para tal é importante atividades de preparação continuada ${ }^{(29) \text {. }}$

De acordo com Os autores ${ }^{(25)}$ a capacidade da enfermagem de responder a desastres é significativa, mas falta uma preparação eficaz. O autor ${ }^{(17)}$ diz que, para responder a um desastre é necessário o hospital e sua equipe está preparada e a falta de simulações realísticas pode ser algum dos motivos para tornar os protocolos ineficazes, pois esta desenvolve um importante papel no preparo.

Portanto os estudos mostram como a atuação do enfermeiro no atendimento perante um incidente com múltiplas vítimas e a necessidade de possuir conhecimentos e habilidades específicas que que precisam ser desenvolvidas através de uma preparação adequada e um planejamento eficaz para garantir o gerenciamento de recursos e triagem fidedigna.

\section{O papel da educação permanente através das simulações realísticas no IMV}

De acordo Os autores ${ }^{(25)}$ apesar da natureza crítica desses eventos, os líderes de enfermagem e administradores recebem pouca educação sobre liderança e tomada de decisão durante um incidente que possui múltiplas vítimas simultaneamente. Uma resposta institucional eficaz requer a integração perfeita de todas as unidades em funcionamento para que a equipe $\mathrm{e}$ os pacientes fiquem protegidos de danos $\mathrm{e}$ garanta a qualidade da assistência. Portanto é essencial o enfermeiro possuir conhecimentos e estarem bem conectados aos demais profissionais que darão suporte a estas vítimas, devido os fatos expostos é importante preparação para uma atuação adequada.

Os autores (16,37) enfatizam que os exercícios de treinamento facilitam o cuidado organizado e define claramente as funções essenciais para raciocínio prático. Para Os autores $^{(24)} \mathrm{o}$ planejamento adequado faz toda a diferença, pois requer a distribuição correta dos recursos, tais como equipamentos da sala de operação, insumos para tratamento de 
lesões e leitos disponíveis para atender as vítimas.

De acordo com os autores ${ }^{(23)}$ a preparação otimiza recursos e facilita o planejamento adequado para o atendimento, pois para este é essencial atender as demandas e desenvolver uma infraestrutura que forneça pessoal, suprimentos, equipamentos e suporte necessário e são os exercícios práticos que fornecem a oportunidade para rever lacunas. Para os autores ${ }^{(35)}$, o gerenciamento e a distribuição correta das vítimas garante a oferta adequada e evita a sobrecarga dos serviços de saúde e o treinamento é essencial para a atuação profissional eficaz, devido a esses fatos ele determina a simulação como um tipo de metodologia ativa no processo de ensino-aprendizado, pois desenvolve pensamento crítico e reflexivo.

Os autores ${ }^{(19)}$ relata que as simulações facilitam o aprendizado e reforça o trabalho em equipe, além de atualizar os profissionais em relação aos protocolos, para os autores ${ }^{(22)}$ essa modalidade de ensino diminui os riscos de falhas. Os autores (30) afirma que a preparação é um instrumento para treinar, avaliar e melhorar o desempenho da equipe dando oportunidades para melhorias.

Os autores ${ }^{(36)}$ ressalta a importância da educação continuada por meio da simulação para um bom planejamento. Os autores ${ }^{(26)}$ afirma que esse tipo de ensino ajuda a enfrentar medos e ansiedade, além de preparar para uma possível atuação com recursos limitados que pode atrapalhar a assistência.

De acordo com o autor ${ }^{(32)}$ as simulações são uma oportunidade de aprender e praticar conhecimentos e melhorar a autoconfiança do profissional. Bentley et $a l .{ }^{(33)}$ cita que esse tipo de exercício melhora a comunicação com a equipe, além de reforçar as habilidades de triagem rápida e precisa, permitindo maximizar o aprendizado preenchendo lacunas entre teoria e a prática.

Em seus estudos, os autores ${ }^{(31,29)}$ ressalta que as simulações são oportunidades de aumentar a autoeficácia, segundo os autores $^{(28)}$ a autoeficácia é a medida em que o indivíduo acredita ser capaz de realizar tarefas específicas, esses exercícios permitem a prática de habilidades e liderança, melhorando a autoconfiança e desempenho, preparando para a tomada de decisão adequada e sendo capaz de gerenciar o surto agudo produzido por um IMV, experiências é uma oportunidade para autoavaliação e permite aos profissionais a familiarize com um sistema de triagem padronizado

Os autores ${ }^{(21,18)}$ declaram que a simulação aumenta a consciência sobre o conhecimento e as habilidades em desastres, melhora a compreensão da responsabilidade da enfermagem, encorajar liderança e o desenvolvimento do trabalho em equipe e contribuem para um feedback construtivo além identificar lacunas, determinar soluções e validar práticas corretas. Da mesma forma, 
o autor ${ }^{(29)}$ enfatiza que o treinamento tem um papel importante na área da saúde e no desempenho ético dos prestadores de serviços e devido a esses aspectos o método é valioso pois faz a adequação do sistema.

Além dos exercícios de simulação $\mathrm{s}$ autores (16,27), afirmam que a troca de experiência se faz importante para obter novos conhecimentos e desenvolver habilidades, além de ser uma oportunidade para refletir sobre as ações, uma boa alternativa para avaliar estresse e desenvolver o preparo psicológico. Para os autores ${ }^{(34)}$ compartilhar sentimentos melhora o desempenho no trabalho em equipe

Os autores ${ }^{(20)}$ afirma que simulações são fardo econômico e por isso torna-se uma barreira crítica para a preparação e resposta ideais, especialmente em pequenos hospitais e instalações de acesso crítico. Porém elas se tornam importantes devido a possibilidade de identificar e corrigir ações em uma assistência emergencial, desenvolvendo a capacidade de determinar com precisão as categorias de triagem.

Diante do exposto dos 22 artigos (100\%) incluídos na revisão e que foram publicados em periódicos de enfermagem em geral, fica claro que as simulações realísticas podem ser utilizadas no processo de ensinoaprendizado pois visam boas práticas de forma que venha agregar e desenvolver novos conhecimentos. Além de ser algo interessante pois prepara os profissionais para possíveis situações direcionando a melhor conduta profissional a ser executada.

\section{Triagem no contexto de incidente com múltiplas vítimas}

Os autores ${ }^{(23)}$ explicam que triagem aos feridos em um IMV é divergente da triagem convencional que ocorre no ambiente hospitalar pois no contexto pré-hospitalar em um IMV concentra no maior bem para o maior número de pessoas, tendo como objetivo identificar rapidamente os pacientes mais graves com a maior chance de sobrevivência usando recursos e tempos menores. Onde a identificação rápida consiste na categorização das vítimas usando codificação de cores como vermelho para agudo e verde para não agudo.

O autor $^{(38)}$ ressalta a importância da triagem em um IMV, pois é através dela que é possível determinar quais pacientes mais precisam de atendimento prioritário de acordo com identificação de ameaças à vida.

$\mathrm{O}$ autor $^{(17)}$ afirma que na triagem inicial das vítimas utiliza-se 4 categorias codificadas por cores para identificar a prioridade e o nível de atendimento necessário. Verde é prioridade III, lesão não grave que requer cuidados menores; amarelo é prioridade II, ferimentos graves que requerem cuidados necessários, mas não imediatos; vermelho é a prioridade I, lesão crítica que requer cuidado imediato; e preto é a 
prioridade 0 , lesão fatal que não requer cuidados.

Os autores (36) acrescenta que os enfermeiros na triagem devem marcar cada vítima com uma etiqueta correspondente ao nível de atendimento, para isso o método START (Simple Triage And Rapid Treatment; Triagem Simples e Tratamento Rápido) que é método de triagem simples e rápido mais utilizado ao redor do mundo, sistematizado que se baseia na capacidade de andar, avaliação da respiração, circulação e nível de consciência após essa triagem os pacientes serão transportados para o local adequado de acordo com suas necessidades seguindo o protocolo de regulamentação.

Diante desses ares torna-se evidente a necessidade de se conhecer os principais parametros de uma triagem para a sistematização da assistencia visando a qualidade e a eficácia. Os dados expostos revelam que o envolvimento dos enfermeiros com a temática em questão é importante e tras a preparação profissional como uma valiosa ferramenta para o atendimento com múltiplas vítimas.

\section{CONSIDERAÇÕES FINAIS}

O estudo sobre a atuação do enfermeiro em um IMV, mostrou que este profissional possui um papel crucial nesse contexto, pois este executa uma importante função no gerenciamento de recursos e na triagem das vítimas envolvidas. Porém existe uma deficiência na preparação destes, iniciando na graduação pela abordagem restrita da temática e pela falta de ensino simulado.

O preparo e treinando dos profissionais serve para adquirir e aprimorar competências e habilidades pois este tem o poder de capacitação para fornecer um atendimento de qualidade nas situações de desastres, visto que o profissional enfermeiro necessita ser criativo no uso de recursos e no gerenciamento da equipe.

\section{REFERENCIAS}

1. Word Health Organization (WHO). Mass casualty management systems: strategies and guidelines for building health sector capacity. Geneva: WHO [Internet] 2007. [Acesso em 17 setembro 2020] Disponivel em: https://apps.who.int/iris/bitstream/handle/106 65/43804/9789241596053_eng.pdf.

2. Brasil. Ministério da Saúde. Secretaria de Atenção à Saúde. Protocolos de intervenção para o SAMU 192. $2^{\mathrm{a}}$ ed. Brasília: Ministério da Saúde [Internet], 2016. [Acesso em: 20 set. 2020]. Disponível em: https://portalarquivos.saude.gov.br/images/pd f/2016/outubro/26/livro-avancado-2016.pdf.

3. Baldi LMC. Atuação de enfermeiro em incidente com múltiplas vítimas. 2015. 57f. Monografia (Conclusão de Curso) Faculdade de Enfermagem Nova Esperança de Mossoró, Rio grande do norte, [Internet] 2015. [Acesso em: 20 set. 2020]. Disponível em:

http://www.sistemasfacenern.com.br/repositor io/admin/acervo/659ca2820dcf1e6a204dfdccd 725e261.pdf.

4. Pertille TS. Direitos Humanos das deslocadas ambientais e os impactos da Usina de Belo 
Monte: a influência internacional nas capacidades humanas centrais. [Internet] 2019. [Acesso em: 20 set. 2020]. Disponível em:

5. Brasil. Lei n. 7.498, de 25 de junho de 1986. Dispõe sobre a regulamentação do exercício da enfermagem e dá outras providências. Diário Oficial da União, Brasília, 26 jun. [Internet] 1986. Seção 1, p. 1. [Acesso em: 20 set. 2020]. Disponível em: http://www.cofen.gov.br/lei-n-749886-de-25de-junho-de-1986_4161.html.

6. Dias A, Gava G. o que é o metodo START? [Internet] 2018. [Acesso em: 20 out. 2020]. Disponível em: www.iespe.com.br/blog/oque-e-o-metodo-start.

7. Intrieri ACU, Barbosa HF, Sabino MRL. et al. O Enfermeiro no APH e o Método Start: uma abordagem de autonomia e excelência. Rev. UNILUS Ensino e Pesquisa, [Internet] mar. 2017;14(34):114-19 [Acesso em: 20 out. 2020]. Disponivel em: http://revista.unilus.edu.br/index.php/ruep/arti cle/view/793/u2017v14n34e793. Acesso em: 30 out. 2020.

8. Oliveira AC, Silva ES, Martuchi SD. Manual do socorrista. $4^{a}$ edição. ISBN: 8581160123, São Paulo. Martinari; 2013.

9. Suport PTL. "Atendimento pré-hospitalar ao traumatizado." Tradução Da $7^{a}$ Edição. Rio De Janeiro. Elsivier Editora; 2012.

10. Aires CMSB. Cuidados de enfermagem especializados perante múltiplas vitimas em situação critica. [Internet] 2015. [Acesso em: 25 out. 2020] Disponível em: http://hdl.handle.net/10400.26/16490.

11. Lima DS, Vasconcelos IF, Queiroz EF, et al. Simulação de incidente com multilas vítimas:treinando rofissionais e ensinando universitários. Rev. Col. Bras Cir [Internet] ago. 2019;46(3) [Acesso em: 10 nov. 2020]. Disponível em: https://doi.org/10.1590/01006991e-20192163.

12. Mendes KDS, Silveira RCCP, Galvão CM.
Revisão integrativa: método de pesquisa para a incorporação de evidências na saúde e na enfermagem. Texto contexto - enferm. [Internet]. Dec 2008;17(4):758-64. [Acesso em: 22 out. 2020]. Disponivel em:https://doi.org/10.1590/S010407072008000400018.

13. Santos AG, Monteiro CFS, Feitosa CDA. et al. Tipos de transtornos mentais não psicóticos em mulheres adultas violentadas por parceiro íntimo: uma revisão integrativa. Rev. esc. enferm. USP [Internet]. 2018;52: e03328. [Acesso em: 22 out. 2020] Disponivel em: https://doi.org/10.1590/s1980220x2017030203328.

14. Polit D F, Beck CT. Fundamentos de pesquisa em enfermagem: avaliação de evidências para a prática da enfermagem. 9. ed. Porto Alegre: Artmed, 2019.

15. Busby S, Witucki-Brown, J. . Theory development for situational awareness in multi-casualty incidents. Journal of Emergency Nursing, [Internet], jan./mar. 2010.37(5):45-58 [Acesso em: 10 nov. 2020].Disponível em: https://pubmed.ncbi.nlm.nih.gov/20234239/.

16. Jong, MJ, Benner, R., Benner, P. de et al. Mass Casualty Care in an Expeditionary Environment: Developing Local Knowledge and Expertise in Context. Journal of Trauma Nursing, [Internet], jan./mar. 2010;17(1):4558 Acesso em: 10 nov. 2020]. Disponível em: https://pubmed.ncbi.nlm.nih.gov/20234239/. Acesso em: 10 nov. 2020.

17. Sloan HM. Responding to a multiple-casualty incident: room for improvement. J Emerg Nurs [Internet]. set. 2011;37(5):484-6 [Acesso em: 10 nov. 2020]. Disponível em: 10.1016/j.jen.2010.07.013.

18. Dasgupta S., French S., Williams-Johnson J. et al. EMS response to an airliner crash. Prehosp Disaster Med., [Internet]. jun. 2012;27(3):299-302 [Acesso em: 30 out. 2020] Disponível em: 10.1017/S1049023X12000854. Acesso em: 30 out. 2020. 
19. Admi H, Eilon Y, Hyams G, Utitz, L. Management of Mass Casualty Events: The Israeli Experience. Journal of Nursing Scholarship, [Internet] 2011;43(2):211-19, [Acesso em: 10 nov. 2020].Disponível em: https://pubmed.ncbi.nlm.nih.gov/21605326/.

20. Glow SD, Colucci VJ, Allington, DR, Noonan CW, Hall EC. Managing multiplecasualty incidents: a rural medical preparedness training assessment. Prehosp Disaster Med., [Internet] ago. 2013;28(4)33441 [Acesso em: 10 nov. 2020]. Disponível em: 10.1017/S1049023X13000423.

21. Shannon CC. Using a simulated mass casualty incident to teach response readiness: a case study. J Nurs Educ., [Internet]. abr. 2015;54(4):215-9. [Acesso em: 10 nov. 2020]. Disponível em: 10.3928/0148483420150318-05.

22. Fletcher L, Justice S, Rohrig L. Designing a disaster. J Trauma Nurs. [Internet] 22(1):3540, jan./fev. 2015. [Acesso em: 10 nov. 2020] Disponível em: 10.1097/JTN.0000000000000098.

23. Vandenberg SL, Scott BD. Preparation for Mass Casualty Incidents. Critical Care Nursing Clinics of North America, [Internet] 2015;27(2):157-66 [Acesso em: 10 out. 2020] Disponível em: https://doi.org/10.1016/j.cnc.2015.02.008.

24. Campion EM, Juillard C, Knudson MM. et al. Reconsidering the Resources Needed for Multiple Casualty Events: Lessons Learned From the Crash of Asiana Airlines Flight 214. JAMA Surg. [Internet].2016;151;6:51217. [Acesso em: 30 out. 2020] Disponível em: 10.1001/jamasurg.2015.5107.

25. Veenema TG, Deruggiero $\mathrm{K}$, Losinski $\mathrm{S}$, Barnett D. Hospital Administration and Nursing Leadership in Disasters An Exploratory Study Using. Health Emergency and Disaster Nursing, [Internet] jul. 2017;4(1):26-38[Acesso em: 30 out. 2020]. Disponível em: https://www.researchgate.net/deref/http\%3A $\% 2 \mathrm{~F} \% 2 \mathrm{Fdx}$.doi.org\%2F10.24298\%2Fhedn.2
015-0016. Acesso em: 30 out. 2020.

26. Hammad KS, Arbon P, Gebbie K, Hutton A. Why a disaster is not just normal business ramped up: Disaster response among ED nurses. Australasian Emergency Care [Internet] 2018;21(1):36-41 [Acesso em: 30 out. 2020]. Disponível em: https://www.sciencedirect.com/science/article /abs/pii/S1574626717300642?via\%3Dihub.

27. Tseng HM, Shih WM, Shen YC, Ho LH, Wu CF. Stress no Trabalho, Resiliência e Qualidade de Vida Profissional entre Enfermeiros que Cuidam de Missas Pacientes vítimas de queimaduras após explosão de poeira colorida Formosa. Journal of Burn Care \& Research, [Internet]. set./out. 2018;39(5):798-804 [Acesso em: 30 out. 2020]. Disponível em: https://doi.org/10.1093/jbcr/irx053.

28. Jonson CO, Pettersson J, Rybing J, Nilsson H, Prytz E. Short simulation exercises to improve emergency department nurses' selfefficacy for initial disaster management: Controlled before and after study. Nurse Education Today [Internet]. 2017;55(201):205, [Acesso em: 30 out. 2020]. Disponível em: https://doi.org/10.1016/j.nedt.2017.04.020.

29. Kiani M, Fadavi M, Khankeh H,Borhani F. Personal factors affecting ethical performance in healthcare workers during disasters and mass casualty incidents in Iran: a qualitative study. Med Health Care Philos, [Internet]. set. 2017;20(3):343-51. [Acesso em: 30 out. 2020]. Disponível em: 10.1007/s11019-0179752-7.

30. Verheul ML, Dückers ML, Visser BB, Beerens RJ, Bierens JJ. Disaster Exercises to Prepare Hospitals for Mass-Casualty Incidents: Does it Contribute to Preparedness or is it Ritualism? Prehospital and Disaster Medicine, [Internet]. 2018;33(4):387-93 [Acesso em: 30 out. 2020]. Disponível em: 10.1017/S1049023X18000584.

31. McElroy JA, Steinberg S, Keller J, Falcone RE. Operation continued care: A large masscasualty, full-scale exercise as a test of 
regional preparedness. Central Surgical Association, [Internet], ago. 2019;166,587-92. [Acesso em: 30 out. 2020]. Disponível em: https://www.surgjournal.com/article/S00396060(19)30330-7/fulltext\#secsectitle0065.

32. Shannon, C. Improving Student Engagement in Community Disaster Preparedness. Journal of Nursing Scholarship, [Internet]. 201944(6):304-07. [Acesso em: 10 nov. 2020] Disponível em: https://journals.lww.com/nurseeducatoronline /Abstract/2019/11000/Improving_Student_En gagement_in_Community_Disaster.10.aspx.

33. Bentley S, Iavicoli L, Boehm L. et al. A simulated mass casualty incident triage exercise: SimWars. Med Ed Portal. [Internet]. 2019;15(10823):1-9. [Acesso em: 30 out. 2020] Disponível em: https://doi.org/10.15766/mep_23748265.10823 .

34. Kim J, Lee O. Effects of a simulation-based education program for nursing students responding to mass casualty incidents: A prepost intervention study. Nurse Education Today [Internet],2020;85 [Acesso em: 30 out. 2020] Disponível em: https://www.sciencedirect.com/science/article /abs/pii/S0260691719308068?via\%3Dihub.

35. Silva RP, Santos VS,Moraes JS, Andrade Í RC, Abreu RNDC, Freitas JG. Aplicabilidade da simulação realística na graduação de enfermagem: experiência em incidentes com múltiplas vítimas. Rev baiana
enferm[Internet] 2020;34:e34648. [Acesso em: 10 nov. 2020]. Disponível em: https://cienciasmedicasbiologicas.ufba.br/inde x.php/enfermagem/article/view/34648.

36. Screws S, Cason M. Mass Casualty Incident Simulation for a Small Space With Limited Resources. Nurs Educ Perspect. [Internet]. set./out. 2020.41(5) [Acesso em: 10 nov. 2020]. Disponível em: 10.1097/01.NEP.0000000000000600.

37. Steen A. Respect for the mass casualty incident in Aurora. J. Emerg. Nurs. [Internet], mar. 2015;41(2):102. [Acesso em: 10 nov. 2020]. Disponível em: 10.1016/j.jen.2014.12.003.

38. Usero-Pérez MDC, Jiménez-Rodríguez ML, González-Aguña A. et al. Validation of an evaluation instrument for responders in tactical casualty care simulations. Revista Latino-Americana de Enfermagem, [Internet]. 2020;28:e3251 [Acesso em: 10 nov. 2020]. Disponível em:10.1590/15188345.3052.3251.

Submissão: 2021-06-24

Aprovado: 2021-07-28

\section{Autor correspondente}

Kályta Sabrina Monteiro de Sousa

Rua Boa Esperança, 1793, Tabajaras 64067475 - Email: kalytasabrina@gmail.com 